\title{
Vinblastine, Bleomycin, and Methotrexate Chemotherapy plus Irradiation for Patients with Early- Stage, Favorable Hodgkin Lymphoma
}

\section{The Experience of the Gruppo Italiano Studio Linfomi}

Paolo G. Gobbi, M.D. ${ }^{1}$

Chiara Broglia, M.D. ${ }^{1}$

Francesco Merli, M.D. ${ }^{2}$

Matteo Dell'Olio, M.D. ${ }^{3}$

Caterina Stelitano, M.D. ${ }^{4}$

Emilio lannitto, M.D. ${ }^{5}$

Massimo Federico, M.D. ${ }^{6}$

Raffaella Bertè, M.D. ${ }^{7}$

Dimitri Luisi, M.D. ${ }^{8}$

Stefano Molica, M... ${ }^{9}$

Carla Cavalli, M.D. ${ }^{10}$

Laura Dezza, M.D. ${ }^{11}$

Edoardo Ascari, м... ${ }^{1}$

${ }^{1}$ Medicina Interna e Oncologia Medica, Università di Pavia, Istituto di Ricovero e Cura a Carattere Scientifico Policlinico San Matteo, Pavia, Italy.

${ }^{2}$ Ematologia, Arcispedale Santa Maria Nuova, Reggio Emilia, Italy.

${ }^{3}$ Ematologia, Istituto di Ricovero e Cura a Carattere Scientifico "Casa Sollievo della Sofferenza," San Giovanni Rotondo, Foggia, Italy.

${ }^{4}$ Ematologia, Ospedali Riuniti, Reggio Calabria, Italy.

${ }^{5}$ Ematologia con Trapianto di Midollo, Università di Palermo, Palermo, Italy.

${ }^{6}$ Oncologia Medica, Università di Modena. Modena, Italy.

${ }^{7}$ Sezione di Ematologia, Divisione di Medicina Interna, Ospedale Civile, Piacenza, Italy.

${ }^{8}$ Oncologia, Ospedale Santo Spirito, Pescara, Italy.

${ }^{9}$ Oncologia, Ospedale A. Pugliese, Catanzaro, Italy.

${ }^{10}$ Medicina Generale, Ospedale Civile, Vigevano, Pavia, Italy.

${ }^{11}$ Medicina II, Ospedale Predabissi, Vizzolo-Predabissi, Italy.

BACKGROUND. The acknowledged effectiveness of vinblastine, bleomycin, and methotrexate (VBM) chemotherapy in patients with early-stage Hodgkin lymphoma has been associated with conflicting toxicity reports.

METHODS. One hundred forty-three patients were evaluated clinically and had favorable Stage IA or IIA Hodgkin lymphoma. Ninety-three patients were treated with the standard VBM schedule combined with extended-field radiotherapy (EFRT), leaving the choice of the therapeutic sequence free. Fifty subsequent patients were treated with a slightly modified VBM schedule (VbMp) combined with RT limited to involved fields (IF-RT) and delivered only after the end of chemotherapy. In the $\mathrm{VbMp}$ schedule, intervals between cycles were 21 days instead of 28 days, bleomycin doses were reduced, small doses of prednisone were given orally, and the interval before RT was prolonged.

RESULTS. Clinical response was complete in $96 \%$ of patients who were treated with VBM plus EF-RT and in $94 \%$ of patients who were treated with VbMp plus IF-RT. Recurrence rates were nearly identical ( $12 \%$ and $11 \%$, respectively) over necessarily different follow-up (91 months and 33 months, respectively). Hematologic toxicity was tolerable in both trials, and pulmonary side effects were moderate in the first trial and negligible in the second. On the whole, treatment was tolerated better when RT followed chemotherapy.

CONCLUSIONS. The VBM regimen was confirmed to be effective in patients with early-stage Hodgkin lymphoma. Administration of all cycles before RT improved tolerance; pulmonary toxicity probably is mitigated further by reduced bleomycin doses, mild prednisone therapy, and a more prolonged resting interval before RT. A slightly higher recurrence rate was expectable in the VBM plus IF-RT trial despite the actual intensification of vinblastine and methotrexate. Cancer 2003;98: 2393-401. (C) 2003 American Cancer Society.

KEYWORDS: Hodgkin, chemotherapy, radiotherapy, toxicity.

here is general consensus on the use of the doxorubicin, bleomycin, vinblastine, and dacarbazine (ABVD) multiple-drug chemotherapy (CT) regimen combined with radiation therapy (RT) in the

Supported by a grant from the Ministero dell'Università (Rome, Italy) and by the Adolfo Ferrata e Edoardo Storti Foundation (Pavia, Italy).

Address for reprints: Paolo G. Gobbi, M.D., Clinica Medica e Oncologia Medica, Università di Pavia, Istituto di Ricovero e Cura a Carattere Scientifico
Policlinico S. Matteo, P. le Golgi no. 2, 27100 Pavia, Italy; Fax: (011) 390382 526223; E-mail: gobbipg@smatteo.pv.it

Received June 3, 2003; revision received August 18, 2003; accepted August 22, 2003. 
management of patients with early-stage Hodgkin lymphoma (HL). ${ }^{1}$ Thus, a variable number of ABVD cycles combined with variably limited irradiation doses and fields are employed in these patients according to more or less favorable clinical presentation. It has been shown that this approach strongly reducesand even abates-the recurrence rate. Subsequent work must focus on reducing toxicity without limiting effectiveness. To this aim, the efficacy of so-called neoadjuvant CT suggests that limited irradiation fields may be used in the combined-modality approach. In contrast, other drug regimens, such as vinblastine, methotrexate, and bleomycin ${ }^{2}$ (VBM); epirubicin, bleomycin, vinblastine, and dacarbazine ${ }^{3}$ (EBVD); and methotrexate, vinblastine, and prednisolone ${ }^{4}$ (MVP), have been tested in combination with RT.

VBM has been adopted in several clinical trials in the last 15 years, and a large amount of evidence of its effectiveness has been collected. However, some concerns still are present regarding its (mainly pulmonary) toxicity due to conflicting results that have emerged from different clinical trials. Because our preliminary experience ${ }^{5}$ with the this regimen in patients with earlystage Hodgkin disease was judged positive, with a favorable balance between effectiveness and toxicity, in 1996, the Gruppo Italiano Studio Linfomi (GISL) decided to close the trial with the standard VBM schedule combined with extended-field RT (EF-RT) and to investigate a therapeutic combination with a few substantial modifications with the objective of further limiting the expected subacute and late toxicity. Changes included completion of the whole CT schedule before RT, intensification of vinblastine and methotrexate with a reduction of the total bleomycin dose, the addition of a mild prednisone medication after every antitumor drug administration, finally, limitation of RT to the involved fields (IF-RT). Intensification of vinblastine and methotrexate was meant to prevent that response from being diminished by restriction of RT, whereas bleomycin reduction and prednisone addition to the CT schedule were intended to mitigate the expected pulmonary toxicity. For the same reason, the interval between the end of CT and the start of RT was prolonged to 4-6 weeks. Herein, we report the results of the new trial, MH- $1 \mathrm{~b}$, which was initiated in 1996, and provide an update on the results of the previous trial, $\mathrm{MH}-1$, in which the accrual was prolonged-after the interim analysis that led to the preliminary report referred to above-until the start of the subsequent MH- $1 \mathrm{~b}$ trial. ${ }^{5}$

\section{MATERIALS AND METHODS}

\section{Patient Populations and Staging Procedures}

The GISL coordinated two subsequent nonrandomized multicenter studies of patients with early-stage, favorable HL: MH-1 started in January 1988 and closed in December 1995, and MH-1b ran from January 1996 until December 2001. The patients on both protocols were treatment-naive, human immunodeficiency virus-negative patients with biopsy-proven Hodgkin disease with a histologic type other than lymphocytedepleted nodular sclerosis or lymphocyte depletion. These patients had clinical Stage IA or IIA HL according to the Cotswolds meeting ${ }^{6}$ criteria and had no more than 3 involved sites, an erythrosedimentation rate $<40 \mathrm{~mm}$ during the first hour, and no bulky masses (according to the Cotswolds recommendations, a tumor mass was considered bulky if it measured $\geq 10 \mathrm{~cm}$ and was located extramediastinally; or, in tumors with a mediastinal location, when its maximum width was equal to or greater than one-third of the internal transverse dimension of the thorax at the level of the fifth and sixth thoracic vertebrae). Due to the planned intensification of 2 of the 3 VBM drugs, inclusion in the $\mathrm{MH}-1 \mathrm{~b}$ trial was restricted to patients younger than age 65 years, and the acceptable lower limit of performance status was defined by a Karnofsky score of 50. Patients with involvement below the diaphragm were not excluded. Two patients with extranodal involvement also were enrolled in the MH-1 study. Informed consent was obtained from each patient.

Staging was strictly clinical and included medical history, physical examination, laboratory tests, chest X-ray examination, abdominal ultrasound, computed tomography scans of chest and abdomen, and unilateral bone marrow core needle biopsy. Lymphangiography was not performed. The primary clinical data on patients from both studies are reported in Table 1. All staging procedures performed at initial assessment were repeated after therapy, with the exception of bone marrow biopsy. A complete remission (CR) was defined as complete regression of measured lesions and the disappearance of all other objective evidence of lymphoma for at least 3 months. Partial remission (PR) consisted of a decrease $>50 \%$ in the sum of the products of the greatest dimensions of measurable lesions. No response (NR) was a decrease $<50 \%$ in measurable lesions; and, finally, progressive disease (PD) was an increase in the size of at least 1 measurable lesion, or the appearance of at least 1 new lesion, or the recurrence of symptoms. ${ }^{6}$

One hundred two patients were included in the MH-1 trial. Three patients with misdiagnosed stage IIIA disease, two patients with mediastinal bulk, three patients with histologic types other than those requested, and one experienced patient were excluded due to protocol violations. Six of 93 eligible patients were older than age 65 years, because there was no age 
TABLE 1

Clinical Staging Characteristics of the Patient Populations in the Two Gruppo Italiano Studio Linfomi Trials ${ }^{\mathrm{a}}$

\begin{tabular}{|c|c|c|c|}
\hline \multirow[b]{2}{*}{ Characteristic } & \multicolumn{2}{|c|}{ MH-1 trial } & \multirow{2}{*}{$\frac{\text { MH-1b trial }}{\text { Age } \leq 65 \text { yrs }}$} \\
\hline & Age $\leq 65$ yrs & Age $>65$ yrs & \\
\hline \multicolumn{4}{|l|}{ Gender (no. of patients) } \\
\hline Male & 57 & 3 & 28 \\
\hline Female & 30 & 3 & 22 \\
\hline \multicolumn{4}{|l|}{ Age (yrs) } \\
\hline Mean \pm SD & $36.9 \pm 13.8$ & $71.8 \pm 3.2$ & $33.5 \pm 11.9$ \\
\hline Range & $16-63$ & $67-79$ & $18-64$ \\
\hline \multicolumn{4}{|l|}{ Karnofsky performance status } \\
\hline Median & 100 & 90 & 100 \\
\hline Range & $70-100$ & $70-100$ & $80-100$ \\
\hline \multicolumn{4}{|l|}{ Stage (no. of patients) } \\
\hline IA & 26 & 2 & 14 \\
\hline $\mathrm{I}_{\mathrm{F}} \mathrm{A}$ & 2 & 2 & 0 \\
\hline IIA & 59 & 2 & 36 \\
\hline \multicolumn{4}{|l|}{$\begin{array}{l}\text { Subdiaphragmatic } \\
\text { presentation }\end{array}$} \\
\hline (no. of patients) & $4 / 87$ & $0 / 6$ & $2 / 50$ \\
\hline \multicolumn{4}{|l|}{ Histology (no. of patients) } \\
\hline $\begin{array}{l}\text { Lymphocyte predominance } \\
\text { (nodular/diffuse) }\end{array}$ & 14 & 1 & 5 \\
\hline \multicolumn{4}{|l|}{ Common variety } \\
\hline $\begin{array}{l}\text { Nodular sclerosis, } \\
\text { undefined }\end{array}$ & 11 & 1 & 7 \\
\hline Nodular sclerosis, cellular & 32 & 0 & 23 \\
\hline Lymphocyte-rich & 3 & 0 & 1 \\
\hline Mixed cellularity & 27 & 4 & 14 \\
\hline \multicolumn{4}{|l|}{ Hemoglobin $(\mathrm{g} / \mathrm{dL})$} \\
\hline Mean \pm SD & $14.0 \pm 1.5$ & $13.4 \pm 0,8$ & $13.5 \pm 1.6$ \\
\hline Range & $11.6-17.5$ & $11.9-14.9$ & $10.0-17.6$ \\
\hline \multicolumn{4}{|l|}{$\begin{array}{l}\text { Lactate dehydrogenase } \\
\qquad(\mathrm{mU} / \mathrm{mL})\end{array}$} \\
\hline Mean \pm SD & $341 \pm 180$ & $333 \pm 84$ & $329 \pm 78$ \\
\hline Range & $103-1449$ & $195-522$ & $140-546$ \\
\hline \multicolumn{4}{|l|}{$\begin{array}{l}\text { Erythrocyte sedimentation rate } \\
\qquad(\mathrm{mm} / \mathrm{hr})\end{array}$} \\
\hline Mean \pm SD & $16.4 \pm 9.8$ & $18.5 \pm 12.5$ & $15.9 \pm 11.8$ \\
\hline Range & $2-37$ & $6-37$ & $2-39$ \\
\hline \multicolumn{4}{|l|}{ Serum albumin (g/dL) } \\
\hline Mean \pm SD & $4.32 \pm 0.44$ & $4.20 \pm 0.52$ & $4.24 \pm 0.51$ \\
\hline Range & $3.49-5.80$ & $3.50-4.70$ & $3.00-5.28$ \\
\hline \multicolumn{4}{|l|}{ Follow-up (mos) } \\
\hline Median & 91 & 59 & 33 \\
\hline Range & $15-170$ & $12-100$ & $6-68$ \\
\hline
\end{tabular}

SD: standard deviation; $\mathrm{mU}$ : milliunits.

${ }^{\text {a }}$ Six elderly patients who were enrolled in the MH-1 trial are listed separately.

restriction in the $\mathrm{MH}-1$ trial; their characteristics are listed separately in Table 1 to allow better comparison of the data on patients from the same age group in both studies. Of the 87 patients age 65 years and younger, 43 patients received RT before VBM, and 41 patients underwent the reversed sequence; 3 patients were treated with 3 CT cycles before and the other 3 CT cycles after interposed irradiation. The median follow-up was 91 months (range, 15-170 months). The preliminary results from the first 50 assessable patients were reported in $1996,{ }^{5}$ with a median follow-up of 38 months.

Fifty-seven patients were enrolled in the $\mathrm{MH}-1 \mathrm{~b}$ study. Fifty patients were assessable for clinical response and toxicity and have been included in this report after a median follow-up of 33 months (range, 6-68 months).

\section{Chemotherapy}

A VBM-based, combined CT-RT schedule was administered in both protocols. In the first trial ( $\mathrm{MH}-1)$, the original VBM regimen associated with EF-IR was adopted, but it left clinicians free to choose the CT and RT sequence in each patient. In the second trial (MH1b), a VBM variant was used (VbMp) with intensified doses of vinblastine and methotrexate and reduced doses of bleomycin-invariably followed by IF-RT.

Both the original VBM schedule and the VbMp schedule are reported in Table 2 . In both trials, the criteria for dose adjustments based on acute hematologic toxicity were the same as the criteria reported by Horning et al. in their first report. ${ }^{2}$ Dose escalations were not allowed. Dose intensity was calculated for each drug and for the whole regimen according to the criteria reported by Hryniuk ${ }^{7}$ and the examples reported by DeVita et al. ${ }^{8}$ According to these calculations, the planned dose intensification in the $\mathrm{VbMp}$ schedule was 1.31 for vinblastine and methotrexate and 0.79 for bleomycin, with an average dose intensity of 1.13 for the whole regimen.

\section{Radiotherapy}

RT was administered by megavoltage equipment in different RT divisions but with different strategies in the two trials. In the MH-1 study, RT consisted of subtotal lymphoid irradiation (STLI), including mantle and paraaortic-splenic pedicle fields for patients with supradiaphragmatic presentation and complete inverted $\mathrm{Y}$ field irradiation (including the spleen) for patients with subdiaphragmatic disease. In the MH-1b study, only involved fields were irradiated, and RT was delivered to one or more of the six lymphatic areas above the diaphragm (Waldeyer ring, bilateral cervical and axillary lymph nodes, mediastinum) or to the five lymphatic areas below in patients with subdiaphragmatic involvement (paraaortic, bilateral iliac, and inguinal). In the two patients with subdiaphragmatic presentation in the $\mathrm{MH}-1 \mathrm{~b}$ trial, the spleen was not involved and, thus, was spared. The boundaries of the irradiation fields were set $5 \mathrm{~cm}$ beyond the areas clinically evaluated as involved. Mediastinal irradiation also included the pulmonary hila with external mar- 
TABLE 2

Schedules of the Two Slightly Different Vinblastine, Bleomycin, and Methotrexate Regimens Used in the Gruppo Italiano Studio Linfomi MH-1 and MH-1b Protocols

\begin{tabular}{|c|c|c|c|c|c|c|c|c|}
\hline \multicolumn{5}{|c|}{ MH-1 protocol: VBM + EF-RT } & \multicolumn{4}{|c|}{ MH-lb protocol: VbMp + IF-RT } \\
\hline Drug & Dose $\left(\mathrm{mg} / \mathrm{m}^{2}\right)$ & Route & $\operatorname{Day}(\mathbf{s})$ & Recycle (days) & Dose $\left(\mathrm{mg} / \mathrm{m}^{2}\right)$ & Route & $\operatorname{Day}(\mathbf{s})$ & Recycle (days) \\
\hline VBL & 6 & I.V. & 1,8 & 28 & 6 & I.V. & 1,8 & 21 \\
\hline Bleo & 10 & I.V. & 1,8 & 28 & $6^{\mathrm{a}}$ or $4^{\mathrm{b}}$ & I.V. & 1,8 & 21 \\
\hline MTX & 30 & I.V. & 1,8 & 28 & 30 & I.V. & 1,8 & 21 \\
\hline Pred & - & - & - & - & $25^{\mathrm{c}}$ & Oral & $1-5,8-12$ & 21 \\
\hline RT & $\mathrm{SLRT}^{\mathrm{d}}(30-40 \mathrm{~Gy})$ & - & - & - & ${\mathrm{IF}-R T^{\mathrm{e}}}^{(30-40 \mathrm{~Gy})}$ & - & - & \\
\hline \multirow{2}{*}{\multicolumn{9}{|c|}{ VBM: vinblastine, bleomycin, and methotrexate; EF-RT: extended-field radiotherapy; VbMp: modified VBM schedule; IF-RT: involved-field radiotherapy; VBL: vinblastine; I.V.: intravenous; Bleo: bleomycin; MTX }} \\
\hline & & & & & & & & \\
\hline \multicolumn{9}{|c|}{ a Cycles 1 and 2.} \\
\hline \multicolumn{9}{|c|}{${ }^{\mathrm{b}}$ Cycles 3-6. } \\
\hline \multicolumn{9}{|c|}{${ }^{\mathrm{c}}$ Fixed dose. } \\
\hline \multicolumn{9}{|c|}{ d SLRT administered before/after/sandwiched between VBM. } \\
\hline${ }^{\mathrm{e}} \mathrm{IF}-\mathrm{RT}$ & stered $25-40$ days after $I$ & & & & & & & \\
\hline
\end{tabular}

gins of $1-2 \mathrm{~cm}$ on healthy lung tissue. In both studies, the recommended doses were $36-40$ grays (Gy), but unavoidable differences in source, energy, dose fraction, total dose, and field area had to be tolerated from institution and institution.

\section{Toxicity Assessment}

Toxicity was measured according to standard Eastern Cooperative Oncology Group (ECOG) ${ }^{9}$ criteria. Due to the preceding experience with VBM, standard chest radiography was the basic test to evaluate and monitor possible pulmonary toxicity from bleomycin and RT. For this purpose, a fine reticular infiltrate at the base of the lungs, alveolar interstitial bibasilar infiltration, progressive lower lobe involvement, and lobar consolidation, respectively, were taken as further grades of toxicity when associated with corresponding symptoms. Pulmonary function tests and measurement of single-breath carbon monoxide diffusing capacity were performed only on demand, when respiratory symptoms (mainly cough and/or dyspnea) were not related to unequivocal lesions on standard chest radiographs.

\section{Statistics}

Toxicity grades were managed statistically as ranks of observations ordered by increasing magnitude and analyzed with the Mann-Whitney $U$ test. ${ }^{10}$ Overall survival (OS) was calculated from the date of diagnosis to the date of last observation or death. Failure-free survival (FFS) was measured from the start of treatment to one of the following events: disease progression during treatment, incomplete remission at the end of treatment, recurrence, or death due to the disease. ${ }^{11}$ A partial remission (PR) and a null response (NR) were considered to be events, because they often hide a resistant or refractory tumor component ${ }^{12}$ and actually share most of the therapeutic requirements ${ }^{13}$ for early recurrences, which currently are considered unquestionable events for statistical purposes. Curves were calculated according to the Kaplan-Meier method, ${ }^{14}$ and deaths due to causes other than HL were not censored.

\section{RESULTS}

The clinical and staging characteristics of the patients in the two studies were very similar (Table 1). Table 3, which reports the responses after combined treatments, shows that there were no differences in clinical outcome between the two protocols nor within the $\mathrm{MH}-1$ trial relative to the adopted CT-RT sequence.

Some differences were observed in the dose intensities of the administered drugs. Relative to the doses planned in the respective schedules, VBM and VbMp had similar dose intensities-0.89 \pm 0.18 (range, 0.47-1.36) and 0.94 \pm 0.11 (range, 0.59-1.15), respectively. Referencing the actual VbMp doses and times of administration to those planned in the original VBM schedule, the mean relative dose intensity of VbMp was $1.07 \pm 0.13$, corresponding to an actual mean intensification of about $20 \%$. This figure results from combining the actual $27-50 \%$ intensification in vinblastine and methotrexate with the $10-22 \%$ decrease in bleomycin dose intensity. Table 4 shows the comparative dose intensity for each drug and for the whole regimen after normalization to the VBM doses; the $\mathrm{MH}-1$ results are grouped further according to whether RT preceded or followed CT. The results dem- 
TABLE 3

Response and Recurrence Rates from the MH-1 Trial and the MH-1b Trial

\begin{tabular}{|c|c|c|c|c|c|c|c|}
\hline \multirow[b]{2}{*}{ Trial } & \multirow[b]{2}{*}{ No. of patients } & \multicolumn{5}{|c|}{ No. of patients } & \multirow[b]{2}{*}{ Median follow-up (mos) } \\
\hline & & CR & PR & NR & PD & Recurrence & \\
\hline \multicolumn{8}{|l|}{ MH-1 } \\
\hline Age $\leq 65$ yrs & 87 & $84(96 \%)$ & 2 & 0 & 1 & $10(12 \%)$ & 91 \\
\hline CT-RT & 41 & 40 & 1 & 0 & 0 & 4 & \\
\hline CT-RT-CT & 3 & 3 & 0 & 0 & 0 & 0 & \\
\hline RT-CT & 43 & 41 & 1 & 0 & 1 & 6 & \\
\hline Age $>65$ yrs & 6 & 6 & 0 & 0 & 0 & 0 & 59 \\
\hline CT-RT & 3 & 3 & 0 & 0 & 0 & - & \\
\hline CT-RT-CT & 3 & 3 & 0 & 0 & 0 & - & \\
\hline MH-lb & 50 & $47(94 \%)$ & 0 & 2 & 1 & $5(11 \%)$ & 33 \\
\hline
\end{tabular}

CR: complete response; PR: partial response; NR: no response; PD: progressive disease; CT: chemotherapy; RT: radiotherapy.

TABLE 4

Dose Intensity of Each Drug and of Whole Administered Regimens (Vinblastine, Bleomycin, and Methotrexate [VBM] and Modified VBM), Normalized to Planned VBM Times and Doses ${ }^{\mathbf{a}}$

\begin{tabular}{lll}
\hline $\begin{array}{l}\text { MH-1: RT + VBM } \\
\text { (43 patients) }\end{array}$ & $\begin{array}{l}\text { MH-1: VBM + RT } \\
\text { (41 patients) }\end{array}$ & $\begin{array}{l}\text { MH-1b: } \\
\text { VbMp + RT } \\
\text { (50 patients) }\end{array}$ \\
\hline VBL & & \\
$0.80 \pm 0.22$ & - & $P<0.0001$ \\
$P=0.0031$ & $0.96 \pm 0.19$ & - \\
- & $P<0.0001$ & $1.22 \pm 0.20$ \\
Bleo & - & \\
$0.82 \pm 0.20$ & $0.96 \pm 0.19$ & - \\
$P=0.0068$ & $P<0.0001$ & $0.74 \pm 0.10$ \\
- & - & $P \leq 0.0001$ \\
MTX & $0.99 \pm 0.17$ & - \\
$0.86 \pm 0.17$ & $P \leq 0.0001$ & $1.25 \pm 0.13$ \\
$P=0.0026$ & & $P \leq 0.0001$ \\
- & - & - \\
Whole regimen (3 drugs) & $0.97 \pm 0.17$ & $1.07 \pm 0.13$ \\
$0.82 \pm 0.17$ & $P=0.0022$ & \\
$P=0.0024$ & & \\
- & &
\end{tabular}

RT: radiotherapy; VBM: vinblastine, bleomycin, and methotrexate; VbMp: modified VBM schedule; VBL: vinblastine; Bleo: bleomycin; MTX: methotrexate.

${ }^{\text {a }}$ Values represent means \pm standard deviations. $P$ values are shown for the differences between the data in the corresponding rows and columns.

onstrate that, in the $\mathrm{MH}-1$ trial, the administered dose intensity of all drugs was significantly higher when CT preceded RT compared with when CT followed RT.

Hematologic toxicity was fully tolerable, as shown in Table 5. The highest toxicity grades (Grades 3-4) were recorded in the MH-1 trial with the RT + CT sequence. In all, the blood cell count was decreased more in the group treated with CT after RT than vice versa. This was demonstrated in the $\mathrm{MH}-1$ trial by the
TABLE 5

World Health Organization Hematologic Toxicity Grades Recorded during Administration of Vinblastine, Bleomycin, and Methotrexate (VBM) or Modified VBM

\begin{tabular}{|c|c|c|c|c|c|c|}
\hline \multirow[b]{2}{*}{ Toxicity } & \multicolumn{5}{|c|}{ Grade (no. of patients) } & \multirow[b]{2}{*}{$P$ value } \\
\hline & 0 & 1 & 2 & 3 & 4 & \\
\hline \multicolumn{7}{|l|}{ Hemoglobin } \\
\hline $\begin{array}{r}\text { EF-RT + VBM } \\
\text { (46 patients) }\end{array}$ & 34 & 7 & 4 & 1 & 0 & $0.081^{\mathrm{a}}$ \\
\hline $\begin{array}{r}\text { VBM + EF-RT } \\
\text { (41 patients) }\end{array}$ & 39 & 2 & 0 & 0 & 0 & $0.209^{\mathrm{b}}$ \\
\hline VbMp + IF-RT & & & & & & \\
\hline (50 patients) & 44 & 5 & 1 & 0 & 0 & $0.555^{\mathrm{c}}$ \\
\hline \multicolumn{7}{|l|}{ Neutrophils } \\
\hline $\begin{array}{r}\text { EF-RT + VBM } \\
\text { (46 patients) }\end{array}$ & 13 & 17 & 9 & 6 & 1 & $>0.001^{\mathrm{a}}$ \\
\hline $\begin{array}{r}\text { VBM + EF-RT } \\
\text { (41 patients) }\end{array}$ & 34 & 4 & 2 & 1 & 0 & $0.105^{\mathrm{b}}$ \\
\hline $\begin{array}{r}\text { VbMp + IF-RT } \\
\text { (50 patients) }\end{array}$ & 32 & 7 & 9 & 2 & 0 & $0.004^{\mathrm{c}}$ \\
\hline \multicolumn{7}{|l|}{ Platelets } \\
\hline $\begin{array}{r}\text { EF-RT + VBM } \\
(46 \text { patients })\end{array}$ & 39 & 2 & 4 & 0 & 1 & $0.385^{\mathrm{a}}$ \\
\hline $\begin{array}{r}\text { VBM + EF-RT } \\
\text { (41 patients) }\end{array}$ & 40 & 1 & 0 & 0 & 0 & $0.105^{\mathrm{b}}$ \\
\hline $\begin{array}{r}\text { VbMp + IF-RT } \\
\text { (50 patients) }\end{array}$ & 47 & 1 & 2 & 0 & 0 & $0.552^{\mathrm{c}}$ \\
\hline $\begin{array}{l}\text { EF-RT: extended-field } \\
\text { field radiotherapy; Vb } \\
\text { a EF-RT + VBM vs. VE } \\
\text { b VBM + EF-RT vs. Vh } \\
{ }^{c} \text { cF-RT + VBM vs. Vb }\end{array}$ & $\begin{array}{l}\text { erapy, } \\
\text { dified } \\
\text {-RT. } \\
\text { F-RT. } \\
\text { F-RT. }\end{array}$ & hed & & & & I: involved- \\
\hline
\end{tabular}

significant difference among grades of neutropenia or by the difference in the grades of anemia according to the adopted CT and RT sequence; an indirect confirmation also comes from the higher doses of myelo- 
TABLE 6

World Health Organization Pulmonary Toxicity Grades Recorded after Different Chemotherapy and Radiotherapy Combinations

\begin{tabular}{|c|c|c|c|c|c|c|}
\hline \multirow[b]{2}{*}{ Toxicity } & \multicolumn{5}{|c|}{ Grade (no. of patients) } & \multirow[b]{2}{*}{$P$ value } \\
\hline & 0 & 1 & 2 & 3 & 4 & \\
\hline \multicolumn{7}{|l|}{$\begin{array}{l}\text { MH-1 trial } \\
\text { EF-RT + VBM }\end{array}$} \\
\hline $\begin{array}{c}\text { EF-RT + VBM } \\
(46 \text { patients }) \\
\text { VBM + EF-RT }\end{array}$ & 39 & 3 & 2 & 2 & 0 & $0.313^{\mathrm{b}}$ \\
\hline (41 patients) & 39 & 1 & 1 & 1 & 0 & - \\
\hline MH-lb trial & & & & & & \\
\hline $\begin{array}{r}\text { VbMp + IF-RT } \\
\text { (50 patients) }\end{array}$ & 48 & 1 & 1 & 0 & 0 & $0.754^{\mathrm{c}}$ \\
\hline $\begin{array}{l}\text { EF-RT: extended-field } \\
\text { field radiotherapy; Vb } \\
\text { a For } 1 \text { vs. } 2+3, P= \\
{ }^{\text {b }} \text { EF-RT + VBM vs. V } \\
{ }^{c} \text { VBM + EF-RT vs. V }\end{array}$ & $\begin{array}{l}\text { herapy } \\
\text { iodifie } \\
\text { for } 1 \\
\text { EF-RT } \\
\text { IF-RT }\end{array}$ & $\begin{array}{l}\text { : vinb } \\
\text { schee } \\
3, P\end{array}$ & , ble & 1, anc & otrexc & RT: involved \\
\hline
\end{tabular}

toxic drugs tolerated in the $\mathrm{MH}-\mathrm{lb}$ trial. It is noteworthy that growth factors never were used prophylactically but were administered only in 7 of 10 patients who exhibited Grade 3 or 4 neutropenia.

Neurologic side effects, to both peripheral nerves and autonomic nerves, were the main nonhematologic toxicities, with no differences observed among the three therapeutic subgroups identified. Defects or full loss of tendon reflexes and difficulty walking were observed in approximately $24 \%$ of patients, but seldom were they severe enough to cause dose modifications. Constipation was more frequent among patients older than age 55 years and, although not absolutely frequent, was responsible for marked drug dose reduction and administration delay. Five patients had to be hospitalized for this problem. Alopecia was rare. Nausea and emesis were controlled well with the use of antiserotoninergic receptor drugs.

Overall, 12 patients experienced respiratory symptoms of different degrees of severity (see Table 6): 7 of 46 patients in the group treated on the EF-RT + VBM schedule, 3 of 41 patients in the group treated on the VBM + EF-RT schedule, and 2 of the 50 patients treated in the VbMp + RT series. Five patients had only cough and mild tachypnea and exhibited fine, reticular lesions on chest X-rays; three of those patients had forced vital capacity and 1-second forced expiratory volume values that were lower than normal. Four other patients exhibited exertional dyspnea with transient cyanosis and fine, bibasilar rales as well as marked radiologic lesions and functional changes (Table 6, Grade 2 column). Three patients experienced severe respiratory failure with persistent hypoxia and thus needed prolonged bed rest. Recovery was com- plete in one patient and was very slow and partial in the other two patients. The differences in frequency and degree of toxicity reported in Table 6 are not statistically significant on their own but become significant when data from the 39 patients treated with the RT + CT schedule are compared with data from the other 87 patients who underwent the reversed sequence, independent of RT field extension and VBM schedule. Moreover, a statistical trend toward lower respiratory side effects in the $\mathrm{MH}-1 \mathrm{~b}$ patients results from the comparison with the $\mathrm{MH}-1$ patients, testing the effect of the whole set of treatment modifications devised in the recent trial (bleomycin reduction, prednisone addition, RT limitation, and prolonged interval between CT and RT). Thus, if administering RT first appears to have greater adverse effects on lungs, despite the fact that the numbers are small, then reduction of the bleomycin dose, the addition of prednisone, and limitation of the irradiated volumes probably contribute to further lower the pulmonary toxicity. Finally, no differences related to smoking or age were observed.

RT was limited to mantle-field RT in 6 patients and to inverted Y field RT in the 4 patients with subdiaphragmatic involvement and consisted of STNI in the remaining 77 patients from the $\mathrm{MH}-1$ trial. In the MH-1b study, RT was delivered strictly to the involved lymphatic areas, which were below the diaphragm in 2 of 50 patients. Daily dose fractions ranged from 1.8 to $2.1 \mathrm{~Gy}$, and total doses actually administered ranged from 35 to $44 \mathrm{~Gy}$ (median, $38.4 \mathrm{~Gy}$ ). No dose boosts were delivered to selected areas. No early toxicity from RT was observed apart from mild myelosuppression in 9 patients (only 2 patients on the $\mathrm{MH}-1 \mathrm{~b}$ protocol) and transient cutaneous changes in 11 patients. No acute pulmonary or cardiac toxicity was recorded during RT. Two secondary neoplasias were recorded, both in patients involved in the $\mathrm{MH}-1$ study lung carcinoma in a nonsmoking woman age 60 -years, who was diagnosed 6 years after the end of treatment, and myelodysplasia in a man age 64 years, 11 years after therapy.

FFS was very similar in both trials, as shown in Figure 1, with a minimal, statistically nonsignificant difference in favor of the $\mathrm{MH}-1$ patients, due to a relatively higher rate of incomplete responses or NRs and an earlier occurrence of recurrences in the $\mathrm{MH}-1 \mathrm{~b}$ trial ( 3 of 50 patients vs. 3 of 87 patients). However, the OS was similar in both trials, as shown in Figure 2, thanks to successful rescue of these patients with second-line therapies.

\section{DISCUSSION}

In the last 15 years, VBM has been considered to be one of the most attractive regimens, in that it fulfills 


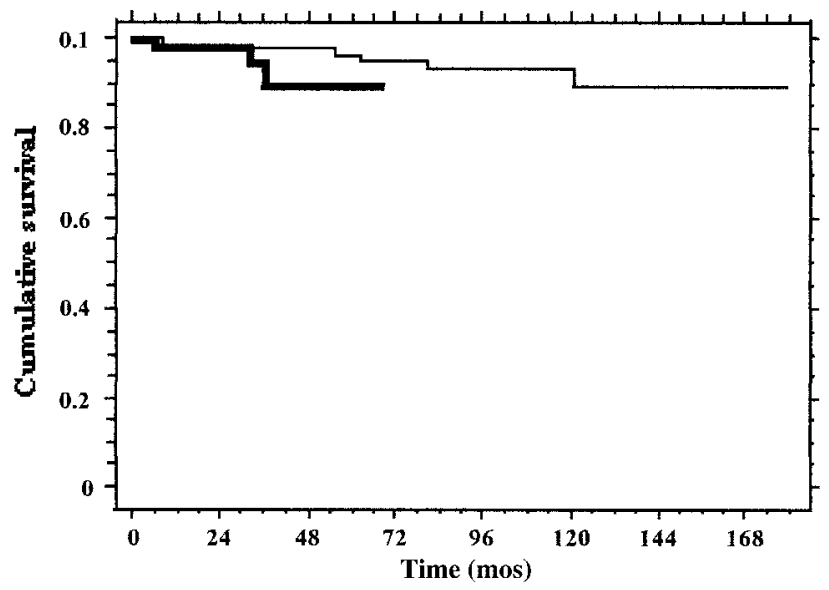

FIGURE 1. Overall survival of patients in the Gruppo Italiano Studio Linfomi MH-1 trial (thin line; $n=87$ ) and the subsequent MH-1b trial (thick line; $n$ $=50 ; P=0.20$ ). Only patients from the $\mathrm{MH}-1$ trial who were of comparable age (i.e., 65 years or younger) were included.

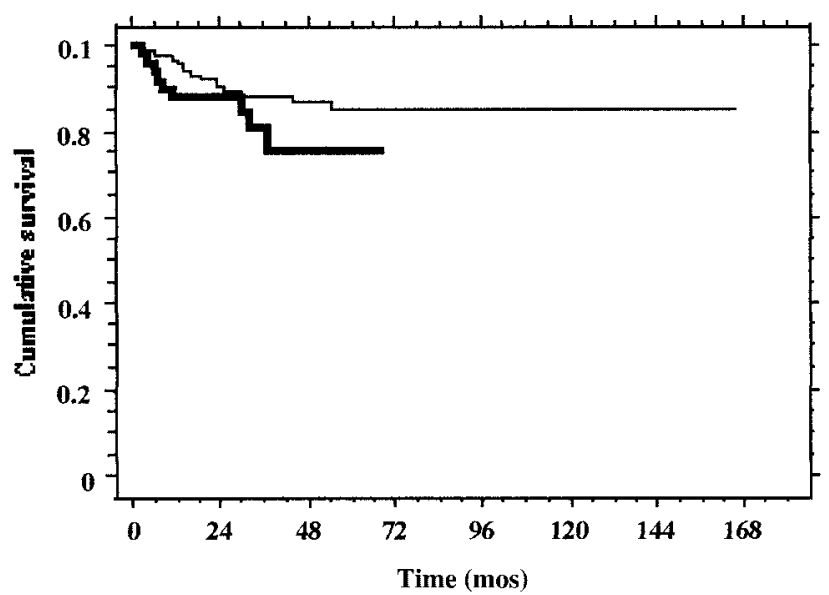

FIGURE 2. Failure-free survival of patients in the Gruppo Italiano Studio Linfomi MH-1 (thin line; $n=87$ ) and $\mathrm{MH}-1 \mathrm{~b}$ trials (thick line; $n=50 ; P$ $=0.18)$.

several requirements of modern treatment for earlystage HL-it avoids surgical staging, reduces the high recurrence rate after RT alone, and spares patients from having to receive alkylating agents and anthracyclines. Indeed, VBM appeared very effective in nearly all of the clinical trials in which it was tested, but conflicting data have been collected regarding its toxicity, probably due to slight (although critical) differences in clinical settings, staging policies, treatment protocols, and endpoints in clinical investigations.

In 1988, Horning et al. $^{2}$ first designed the VBM schedule and demonstrated that in 67 patients with surgically staged HL, including pathologic Stages IA to IIIA, the effectiveness of such a CT regimen was able to compensate for limiting RT to the involved fields only.
FFS rates at 5 years were $70 \%$ in the subtotal or total lymph node irradiation arm $(n=35)$ and $95 \%$ in the arm treated with IF-RT plus VBM $(n=32)$. Hematologic and pulmonary toxicities were acceptable. It was concluded that in patients with laparotomically staged disease, a significant reduction in the irradiated volumes could be counterbalanced fully by subsequent monthly administration of six cycles of VBM CT.

In 1994, the British National Lymphoma Investigation (BNLI) ${ }^{15}$ reported on a study involving patients with clinical Stage IA and IIA disease who were treated with IF-RT plus VBM CT. In that study, CT was intended to counterbalance both possible staging inaccuracies and reductions in RT. The CR rate was $100 \%$, and only 2 of 30 patients (7\%) developed recurrent disease. Toxicity, both hematologic and pulmonary, was severe, and the regimen was judged to be unsuitable for routine use. It is noteworthy that the BNLI used a sandwich protocol in which two VBM cycles were delivered before RT and four cycles were delivered after RT.

In 1996, the GISL ${ }^{5}$ published the preliminary results of the MH-1 protocol for patients with clinical Stage IA and IIA disease whose final results are reported in the current paper. The protocol was based on EF-RT and VBM CT, with no age restriction for elderly patients. The objective of the study was to assess whether the VBM regimen could mitigate the expectable inaccuracy of a merely clinical staging (also without lymphangiography). Effectiveness was high in the first 50 patients treated ( $100 \%$ CR rate with a total of 5 recurrences). VBM was administered after RT in 31 patients and before RT in 13, whereas 6 patients received a sandwich treatment, with 3 cycles before RT and 3 cycles after RT. The schedule recycled every 28 days, and the mean dose intensity actually delivered was $0.82 \pm 0.18$. Less toxicity was observed compared with the BNLI study, and the cycles appeared to be better tolerated when they were administered before RT than when they were administered after RT or in the sandwich schedule. In particular, the study demonstrated that pulmonary toxicity was related primarily to the total dose of mediastinal irradiation and secondarily to the total dose of bleomycin administered.

A subsequent randomized study by Horning et al. ${ }^{16}$ investigated the ability of the VBM regimen combined with regional RT to balance the reduction in RT from extended fields to involved fields in 78 patients with clinical Stage IA and IIA disease. All patients experienced $\mathrm{CR}$, and the FFS rate was $92 \%$ for patients who were treated with STLI alone and $93 \%$ for patients who received VBM plus regional RT. In this group, RT was sandwiched between the first two VBM cycles and the last four VBM cycles. In the last 4 cycles, the bleomycin dose was decreased from $10 \mathrm{mg} / \mathrm{m}^{2}$ to $2.5 \mathrm{mg} / \mathrm{m}^{2}$. He- 
matologic toxicity was mild, and six patients had radiologic signs of radiation pneumonia. Although specific data on the pulmonary toxicity recorded in this study were not given but were cumulated with those from other combination therapies, the authors concluded that there was an inherent risk in combining major doses of two potential pulmonary toxins-irradiation and bleomycin. Thus, they did not advocate the routine use of combined VBM and RT in clinical practice.

In 2000, Zinzani et al. ${ }^{17}$ reported the results of a pilot study on 19 clinically evaluated elderly patients (median age, 68 years; age range, 60-75 years) with different stages of disease; the regimen consisted of VBM CT combined with very limited RT that was delivered only to the initial bulky mass after the completion of CT. Fifteen patients responded completely, 3 (all with advanced-stage disease) responded only partially, and 1 did not respond at all. Two of three patients who developed recurrent disease achieved a second CR with a different CT line. Five patients died, all of their disease. Such results, which have to be considered satisfactory in an elderly patient setting, were obtained with negligible toxicity: mild hematologic side effects were observed in $30 \%$ of patients, and severe side effects ( $\geq$ ECOG Grade 3) were observed in only 1 patient. Transfusions, dose reductions, and administration of growth factors never were required. No pulmonary or cardiac toxicity was recorded, not even in patients who received mediastinal irradiation. The authors concluded that VBM is a safe and effective therapeutic option for elderly patients.

The $\mathrm{BNLI}^{4}$ prolonged its experience with VBM by treating another 30 early-stage, asymptomatic patients with intermediate risk according to a specific prognostic index. Patients were staged clinically and received RT only to initially involved areas after the first two VBM cycles and before the last four VBM cycles. All patients responded completely, five patients subsequently developed recurrent disease, and four patients were rescued with different salvage treatments: one patient died of disease. The VBM regimen also caused marked acute hematologic toxicity in this second BNLI trial, and acute pulmonary toxicity affected $47 \%$ of patients: thus, VBM was abandoned definitively.

Finally, Martinelli et al. ${ }^{18}$ reported very good results with a sandwich protocol delivering 3 VBM cycles followed by IF-RT and then by 4 more VBM cycles in which the bleomycin dose was reduced from 10 $\mathrm{mg} / \mathrm{m}^{2}$ to $6 \mathrm{mg} / \mathrm{m}^{2}$. Patients had clinical Stage IA or IIA disease and were monitored carefully with regard to pulmonary function. Forty-two of 43 patients responded completely, 7 patients developed recurrent disease within the first 3 years, and 5 patients were rescued with alternative treatments. No patients experienced acute or late pulmonary toxicity. The authors consider such a protocol effective and safe for patients with early-stage disease; their only concern was the length of the whole treatment.

Thus, with reference to surgical staging and EFRT, which was the standard therapy for patients with favorable, early-stage HL 15 years ago, some studies tested VBM CT only to reduce the extension of RT, ${ }^{2}$ others simply to avoid surgical staging (GISL-MH1 trial; see Gobbi et al. ${ }^{5}$ and current data), others for both purposes in either young patients ${ }^{4,15,16,18}$ (GISL $\mathrm{MH}-1 \mathrm{~b}$ trial; current report) or elderly patients, ${ }^{17}$ or even in both. ${ }^{5}$ All studies had excellent response rates ranging from $94 \%$ to $100 \%$ in younger patients and from $79 \%$ to $100 \%$ in elderly patients. Pulmonary toxicity was a major problem in some (but not all) of those studies. Indeed, pulmonary toxicity was observed when either the whole VBM regimen or at least some cycles were administered after RT, ${ }^{2,4,15,16}$ as in the sandwich combination. The so-called sandwich schedule for delivering combined-modality therapy was used commonly in the late 1970s and early 1980s and then slowly was abandoned because of heavier hematologic toxicity experienced in the cycles delivered after RT. The first investigators who pointed out the better tolerability of CT before RT were Kun et al., ${ }^{19}$ for mechlorethamine, vincristine, procarbazine, and prednisone (MOPP), and Bonadonna et al., ${ }^{20}$ for ABVD. The results of the current study appear to confirm this observation as it relates to VBM, probably due to a more toxic effect of drugs on bone marrow that became hyperplastic after RT. It is noteworthy that in the available reports on VBM therapy, pulmonary toxicity generally was judged mild and tolerable, independent of age, in the patients who received RT after completion of the CT program ${ }^{5,17}$ (see also the current data). In addition, the length of the rest period between CT and RT may play a role in the development of pulmonary toxicity, as demonstrated for hematologic toxicity. In the GISL experience, the interval between the end of CT and the start of RT was 26 days \pm 8 days in the MH-1 trial for patients who completed the CT program before RT and 34 days \pm 9 days in the corresponding patients in the $\mathrm{MH}-1 \mathrm{~b}$ trial. It is worth remembering that in patients with HL, the first combined treatment protocols ${ }^{21}$-which delivered variable numbers of MOPP cycles after irradiation-recommended resting periods of 6-8 to 8-12 weeks before RT. The Milan experience with ABVD started ${ }^{20}$ with a resting period of 4-6 weeks between $A B V D$, and it is noteworthy that hematologic tolerability was improved and that there was no alarming pulmonary toxicity, despite the use of the same bleomycin doses used in standard VBM and the administration of mantle irradiation to nearly all patients. It is questionable whether the reduction of bleomycin in the 
last CT cycles truly can help to decrease pulmonary toxicity: bleomycin doses were reduced in the $\mathrm{MH}-1 \mathrm{~b}$ GISL trial and in the study by Martinelli et al. ${ }^{18}$ (both with exactly the same scheduled doses), in which pulmonary toxicity was infrequent, whereas no advantage in terms of lung tolerance was observed in the StanfordKaiser Permanente experience, even after a greater reduction $\left(2.5 \mathrm{mg} / \mathrm{m}^{2}\right)$ in the last 4 cycles administered after RT.

Apart from toxicity, there is a distinct concern regarding the effectiveness of the whole combined schedule of modified VBM (VbMp) followed by IF-RT. Although the CR rate (94\%) may be considered acceptable in the modern management of patients with HL, the observed recurrence rate is not fully satisfactory, with a mild excess in terms of early recurrences compared with the preceding $\mathrm{MH}-1$ experience. The increased dose intensity of the administered $\mathrm{VbMp}$ may not have been able to counterbalance fully the reduction of irradiation to strictly involved sites, which resulted in a slightly increased recurrence rate compared with VBM + EF-RT. More prolonged follow-up will be necessary to understand the actual trend of the remission duration curve and to design further modifications in the treatment schedule. The use of a hypothetical CT regimen that does not contain alkylating agents, anthracyclines, or bleomycin combined with IF-RT appears to be the current challenge in the treatment of patients with earlystage HL to lower the risk of second malignancies, cardiac incidents, and pulmonary toxicity. From this point of view, the ABVD regimen itself, in which anthracycline and bleomycin often are combined with RT, can only be considered the current gold standard, and not an absolute ideal. Future efforts should be aimed at designing a more effective, less toxic CT regimen, because further reductions in irradiation doses and fields for the frequently involved mediastinum in these patients are hardly feasible. Along with the intrinsic effects of bleomycin on the lungs, both vinblastine and methotrexate have been reported as factors that potentially enhance the pulmonary effects of irradiation ${ }^{22}$ and can be considered for possible changes in the treatment schedule. Some drugs, like vinorelbine and gemcitabine, also can be considered as interesting additional or replacement agents in the VBM schedule to come near the optimal CT schedule for the combined treatment of patients with early-stage HL.

\section{REFERENCES}

1. Aisenberg AC. Problems in Hodgkin's disease management. Blood. 1999;93:761-779.

2. Horning SJ, Hoppe RT, Hancock SL, et al. Vinblastine, bleomycin, and methotrexate: an effective adjuvant chemotherapy in favorable Hodgkin's disease. J Clin Oncol. 1988;6:1822-1831.

3. Nordijk E, Carde P, Mandard AM, et al. Preliminary results of the EORTC-GPMC controlled clinical trial $\mathrm{H} 7$ in early stage Hodgkin's disease. EORTC Lymphoma Cooperative Group. Group Pierre-et-Marie-Curie. Ann Oncol. 1994; 5(Suppl 2):523-529.

4. Moody AM, Pratt J, Hudson GV, et al. British National Lymphoma Investigation: pilot studies of neoadjuvant chemotherapy in clinical Stage IA and IIA Hodgkin's disease. Clin Oncol (R Coll Radiol). 2001;13:262-268.

5. Gobbi PG, Pieresca C, Frassoldati A, et al. Vinblastine, bleomycin, and methotrexate chemotherapy plus extended-field radiotherapy in early, favorably presenting, clinically staged Hodgkin's patients: the Gruppo Italiano per lo Studio dei Linfomi experience. J Clin Oncol. 1996;14:527-533.

6. Lister TA, Crowther D, Sutcliff SB, et al. Report of a committee convened to discuss the evaluation and staging of patients with Hodgkin's disease. J Clin Oncol. 1989;7:1630-1636.

7. Hryniuk WM. Average relative dose intensity and the impact on design of clinical trials. Semin Oncol. 1987;14:65-74.

8. DeVita VT Jr., Hubbard SM, Longo DL. The chemotherapy of lymphoma: looking back, moving forward-The Richard and Hinda Rosenthal Foundation Award Lecture. Cancer Res. 1987;47:5810-5824.

9. Oken MM, Creech RH, Tormey DC, et al. Toxicity and response criteria of the Eastern Cooperative Oncology Group. Am J Clin Oncol. 1982;5:129-137.

10. Armitage P, Berry G. Statistical methods in medical research (2nd edition). Oxford: Blackwell Scientific, 1987:296-357.

11. Dixon DO, McLauglin P, Hagemeister FB, et al. Reporting outcomes in Hodgkin's disease and lymphoma. J Clin Oncol. 1987;5:1670-1672.

12. Carde P. Should poor risk patients with Hodgkin's disease be sorted out for intensive treatments? Leuk Lymphoma. 1995;15:31-40.

13. Longo DL, Duffey PL, Young RC, et al. Conventional-dose salvage combination chemotherapy in patients relapsing with Hodgkin's disease after combination chemotherapy: the low probability for cure. J Clin Oncol. 1992;10:210-218.

14. Kaplan EL, Meier P. Nonparametric estimation from incomplete observation. J Am Stat Assoc. 1958;53:457-481.

15. Bates NP, Williams MV, Bessell EM, et al. Efficacy and toxicity of vinblastine, bleomycin, and methotrexate with involved-field radiotherapy in clinical Stage IA and IIA Hodgkin's disease: a British National Lymphoma Investigation pilot study. J Clin Oncol. 1994;12:288-296.

16. Horning SJ, Hoppe RT, Mason J, et al. Stanford-Kaiser Permanente G1 study for clinical Stage I to IIA Hodgkin's disease: subtotal lymphoid irradiation versus vinblastine, methotrexate and bleomycin chemotherapy and regional irradiation. J Clin Oncol. 1997;15:1736-1744.

17. Zinzani PL, Magagnoli M, Bendandi M, et al. Efficacy of the VBM regimen in the treatment of elderly patients with Hodgkin's disease. Haematologica. 2000;85:729-732.

18. Martinelli G, Cocorocchio E, Peccatori F, et al. Abstract CO-024 [abstract]. Haematologica. 2001;86(Suppl 10):19.

19. Kun LE, DeVita VT Jr., Young RC, et al. Treatment of Hodgkin's disease using intensive chemotherapy followed by irradiation. Int J Radiat Oncol Biol Phys. 1976;1:619-626.

20. Bonadonna G, Zucali R, De Lena M, et al. Combined chemotherapy (MOPP or ABVD)-radiotherapy approach in advanced Hodgkin's disease. Cancer Treat Rep. 1977;61:769-777.

21. Kaplan HS, Rosenberg SA. The management of Hodgkin's disease. Cancer. 1975;36:796-803.

22. Ginsberg SJ, Comis R. The pulmonary toxicity of antineoplastic agents. Semin Oncol. 1982;9:34-42. 